\title{
Bacterial communities in the digester bed and liquid effluent of a microflush composting toilet system
}

\author{
Laura E Williams ${ }^{\text {Corresp., }}{ }^{1}$, Claire E Kleinschmidt ${ }^{1,2}$, Stephen Mecca ${ }^{2}$ \\ 1 Department of Biology, Providence College, Providence, RI, USA \\ 2 Department of Engineering-Physics-Systems, Providence College, Providence, RI, USA \\ Corresponding Author: Laura E Williams \\ Email address: Iwillia7@providence.edu
}

Lack of access to clean water and sanitation is a major factor impacting public health in communities worldwide. To address this, the S-Lab at Providence College and the Global Sustainable Aid Project developed a microflush composting toilet system to isolate and treat human waste. Solid waste is composted within a filter-digester bed via an aerobic process involving microbes and invertebrates. Liquid waste may be sanitized by solar disinfection (SODIS) or slow sand filtration (SSF). Here, we used 16S rRNA amplicon sequencing of samples from a scaled-down test version of the system to better understand the bacterial component of the toilet system. Immediately after fecal matter was deposited in the test system, the bacterial community of the filter-digester bed at the site of deposition resembled that of the human gut at both the phylum and genus level, which was expected. Genus-level analysis of filter-digester bed samples collected over the next 30 days from the site of deposition showed reduced or undetectable levels of fecalassociated taxa, with the exception of Clostridium XI, which persisted at low abundance throughout the sampling period. Starting with the sample collected on day 4 , the bacterial community of the filter-digester bed at the site of deposition was dominated by bacterial taxa commonly associated with environmental sources, reflecting a major shift in bacterial community composition. These data support the toilet system's capacity for processing solid human waste. We also analyzed how SODIS and SSF sanitization methods affected the bacterial community composition of liquid effluent collected on day 15 from the test system. Untreated and treated liquid effluent samples were dominated by Proteobacteria. At the genus level, the bacterial community of the untreated effluent included taxa commonly associated with environmental sources. In the SODIS-treated effluent, these genera increased in abundance, whereas in the SSF-treated effluent, they were greatly reduced or undetectable. By analyzing OTUs that were unclassified at the genus level, we observed that SSF appears to introduce new taxa into the treated effluent, likely from the biological film of microbes and small animals that constitutes the key element of slow sand 
filtration. These data will inform continued development of liquid waste handling strategies for the toilet system. Using the test system as an indicator of the performance of the fullscale version, we have shown the effectiveness of the microflush composting toilet system for containing and eliminating gut-associated bacteria, thereby improving sanitation and contributing to better public health in rural and peri-urban communities. 
1

2

3

4

5

6

7

8

9

10

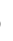

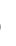

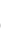

Bacterial communities in the digester bed and liquid effluent

of a microflush composting toilet system

Laura E. Williams ${ }^{1 *}$, Claire E. Kleinschmidt ${ }^{1,2}$, Stephen Mecca $^{2 \dagger}$

${ }^{1}$ Department of Biology, Providence College, Providence, RI

${ }^{2}$ Department of Engineering-Physics-Systems, Providence College, Providence, RI

$\dagger$ Deceased

9

*Corresponding author:1willia7@providence.edu 


\section{Abstract}

12 Lack of access to clean water and sanitation is a major factor impacting public health in communities worldwide. To address this, the S-Lab at Providence College and the Global

Sustainable Aid Project developed a microflush composting toilet system to isolate and treat human waste. Solid waste is composted within a filter-digester bed via an aerobic process involving microbes and invertebrates. Liquid waste may be sanitized by solar disinfection

17 (SODIS) or slow sand filtration (SSF). Here, we used 16S rRNA amplicon sequencing of samples from a scaled-down test version of the system to better understand the bacterial component of the toilet system. Immediately after fecal matter was deposited in the test system, the bacterial community of the filter-digester bed at the site of deposition resembled that of the

21 human gut at both the phylum and genus level, which was expected. Genus-level analysis of 22 filter-digester bed samples collected over the next 30 days from the site of deposition showed 23 reduced or undetectable levels of fecal-associated taxa, with the exception of Clostridium XI, 24 which persisted at low abundance throughout the sampling period. Starting with the sample 25 collected on day 4, the bacterial community of the filter-digester bed at the site of deposition was 26 dominated by bacterial taxa commonly associated with environmental sources, reflecting a major 27 shift in bacterial community composition. These data support the toilet system's capacity for 28 processing solid human waste. We also analyzed how SODIS and SSF sanitization methods 29 affected the bacterial community composition of liquid effluent collected on day 15 from the test 30 system. Untreated and treated liquid effluent samples were dominated by Proteobacteria. At the 31 genus level, the bacterial community of the untreated effluent included taxa commonly

32 associated with environmental sources. In the SODIS-treated effluent, these genera increased in 33 abundance, whereas in the SSF-treated effluent, they were greatly reduced or undetectable. By 
34 analyzing OTUs that were unclassified at the genus level, we observed that SSF appears to 35 introduce new taxa into the treated effluent, likely from the biological film of microbes and small

36 animals that constitutes the key element of slow sand filtration. These data will inform continued

37 development of liquid waste handling strategies for the toilet system. Using the test system as an

38 indicator of the performance of the full-scale version, we have shown the effectiveness of the

39 microflush composting toilet system for containing and eliminating gut-associated bacteria,

40 thereby improving sanitation and contributing to better public health in rural and peri-urban

41 communities.

42

43 Introduction

44 In 2015, the United Nations released a global sustainable development agenda featuring 17 goals

45 with specific outcomes to alleviate poverty, improve public health and strengthen environmental

46 protection by 2030 (UN General Assembly, 2015). In particular, Goal 6 is focused on providing

47 clean water and sanitation to communities around the world. Water and sanitation directly impact

48 health and wellbeing, yet 2.1 billion people lack safely managed sources for drinking water, and

492.3 billion people lack access to basic sanitation facilities such as toilets (World Health

50 Organization and United Nations Children's Fund, 2017). This disparity results in a heavy

51 disease burden on affected communities. According to the World Health Organization, 870,000

52 people died in 2016 due to inadequate sanitation and unsafe drinking water (World Health

53 Organization, 2018). Isolating and treating human waste is one strategy to reduce contamination

54 of water sources and improve public health. 
56 To this end, the S-Lab at Providence College and the Global Sustainable Aid Project (GSAP)

57 developed a low cost, microflush toilet system that provides an effective sanitation solution for

58 rural and peri-urban areas (Mecca et al., 2013a). The GSAP Microflush toilet is designed to use

59 locally sourced materials so that a trained local artisan can fabricate the system. The user

60 interface sits above a microflush valve, which uses the small volume $(\sim 150 \mathrm{cc})$ of greywater

61 from the previous user's hand wash to move both solid and liquid waste into a filter-digester bed.

62 The bed measures 3' $\times 6^{\prime}$ for the household version of the toilet and 4' $\times 7^{\prime}$ ' for a larger school

63 block version. Liquid waste flows through a series of screens and mesh to a filtrate chamber that

64 lies below, whereas solids are digested and composted in the layered bed by an aerobic process

65 involving microbes and invertebrates (usually the earthworm Eisenia fetida). The rear cover of

66 the GSAP toilet can be removed to retrieve the compost every 2-3 years for use in agriculture.

67

68 Liquid effluent typically exits the filtrate chamber of the GSAP Microflush toilet via a drainage

69 pipe to a soak hole, which acts as a scaled-down version of a leach field. For communities with

70 high water tables, there are two options under consideration for sanitization of liquid effluent:

71 solar disinfection (SODIS) or slow sand filter (SSF). SODIS involves collecting liquid effluent in

72 a clear container such as a recycled water bottle and exposing the container to sunlight. This

73 approach significantly reduces levels of bacteria, including pathogens (Boyle et al., 2008;

74 Caslake et al., 2004). SSF treatment involves migration of liquid effluent through a naturally

75 formed biological film (termed the schmutzdecke) containing microbes and sometimes small

76 animals. This film, which sits atop layers of sand and gravel, removes pathogens and other

77 contaminants (Elliott et al., 2008; Hijnen et al., 2004). 
79 The ability of the GSAP Microflush toilet to process waste is supported by previous studies

80 investigating temperature distribution within the digester (Mecca et al., 2017) and the use of

81 SODIS for pathogen reduction in liquid effluent (Mecca et al., 2013b). In addition, studies of

82 vermicomposting as a waste treatment method have demonstrated the effectiveness of this

83 approach for mass reduction and pathogen removal (Hill and Baldwin, 2012; Soobhany et al.,

84 2017). However, little is known about how the bacterial community composition of the filter-

85 digester bed within the GSAP Microflush toilet changes over time after waste is deposited or

86 how SODIS and SSF treatment impact the bacterial community composition of liquid effluent.

87 To investigate these questions, we analyzed samples from a test system (Figure 1), which is a

88 scaled down version of the field system described above. The test system had been maintained

89 for over three years by adding human fecal matter and vegetable scraps multiple times per week,

90 which simulated the use of the field system.

91

92 Using 16S rRNA amplicon sequencing, we analyzed the bacterial community composition of the

93 filter-digester bed immediately after depositing human fecal matter, and we then sampled the

94 filter-digester bed four times over the next 30 days to track the abundance of fecal-associated

95 OTUs and assess changes in community composition. We also compared the bacterial

96 community composition of untreated liquid effluent collected from the filtrate chamber on day

9715 with samples of the same effluent treated by either SODIS or SSF to investigate the effects of

98 these sanitization methods. These data yield insight into the bacterial component of the GSAP

99 Microflush toilet system and will help inform further development and deployment of this

100 valuable sanitation solution.

101 
102 Materials and Methods

103 Filter-digester bed sample collection

104 At time zero, human fecal matter was deposited in the filter-digester bed of the test system, then

105 a sample of this fecal matter was taken with a sterile spoon and transferred to a sterile $50 \mathrm{ml}$

106 conical tube. No additional materials were added to the test system for the duration of the

107 experiment. On days $4,10,15$, and 30 , a sterile spoon was used to mix the material at the 108 location of the initial deposit, and a sample was then transferred to a sterile $50 \mathrm{ml}$ conical tube.

109 All samples were taken at approximately the same time of day and placed at $-80^{\circ} \mathrm{C}$ within a few 110 hours.

111

112 Liquid effluent sample collection and treatment

113 On day 15, liquid effluent was collected from the drainage pipe connected to the filtrate chamber

114 at the base of the test system. This 15-day period allowed time for liquid waste in the filter-

115 digester bed to migrate through the layered bed and the series of screens and mesh separating the

116 bed and the filtrate chamber. Samples of the collected effluent were transferred to sterile $500 \mathrm{ml}$

117 sample bottles and stored at $-80^{\circ} \mathrm{C}$ as raw (untreated) liquid effluent. The remainder of the

118 effluent was used to test two different methods for sanitizing liquid effluent. To test the Solar

119 Disinfection (SODIS) method, liquid effluent was immediately poured into a non-sterile Platypus

120 bag, which was then attached to a solar rack at a $45^{\circ}$ angle and exposed to direct sunlight. After

12124 hours, a sample of the treated effluent was transferred to a sterile $500 \mathrm{ml}$ sample bottle and

122 stored at $-80^{\circ} \mathrm{C}$. To test the Slow Sand Filter (SSF), liquid effluent was transported to the lab in 5

123 gallon closed containers and stored at ambient temperature for $<24$ hours. To begin the filtration

124 process, $9 \mathrm{~L}$ of water was pushed through the filter to fill the chamber. Then, $3 \mathrm{~L}$ of liquid 
125 effluent was pushed through the filter, followed by $33 \mathrm{~L}$ of water. Considering the exit rate and

126 void volume of the SSF, we calculated that the liquid effluent would exit the system after 10

127 hours. In addition, the liquid effluent fraction was visibly different from the water fractions,

128 which enabled us to take a sample midway through the liquid effluent fraction exiting the

129 system. The sample was collected with a sterile $500 \mathrm{ml}$ sample bottle and stored at $-80^{\circ} \mathrm{C}$.

131 Genomic DNA extraction

132 Genomic DNA was extracted from the filter-digester bed and liquid effluent samples using the

133 PowerSoil DNA Isolation Kit (MO BIO Laboratories, Carlsbad, CA). For the filter-digester bed

134 samples, 0.26-0.28 g of sample were used. For the liquid effluent samples, samples were thawed

135 and then centrifuged in sterile centrifuge bottles at $4000 \mathrm{rpm}$ for 15 minutes in two $250 \mathrm{ml}$

136 increments to pellet bacterial cells and other solid particulates. Pellets were transferred to the

137 PowerSoil tubes to begin the extraction process. All filter-digester bed and liquid effluent

138 samples were processed together in one run, along with a negative kit control to test for bacterial

139 DNA contamination in the genomic DNA extraction kit. This negative kit control was a single

140 prep taken through all of the steps alongside the samples but with no sample added at the

141 beginning. Genomic DNA concentration was estimated using a Qubit analyzer. The genomic

142 DNA extract with the lowest concentration was estimated at $3.77 \mu \mathrm{g} / \mathrm{mL}$. Each genomic DNA

143 extract was diluted to this concentration using PCR grade water (MO BIO Laboratories,

144 Carlsbad, CA). Genomic DNA extracts and dilutions were stored at $-20^{\circ} \mathrm{C}$.

145

$14616 S$ rRNA gene amplification 
147 Using the dilutions of genomic DNA as template, we amplified the V4 region of the 16S rRNA

148 gene following a two-step protocol. We performed the initial amplification step with KAPA HiFi

149 HotStart Ready Mix using primers 515F and 806R (Caporaso et al., 2011) modified with

150 overhang adapters (515F_mod:

151 TCGTCGGCAGCGTCAGATGTGTATAAGAGACAGGTGCCAGCMGCCGCGGTAA,

152 806R_mod:

153 GTCTCGTGGGCTCGGAGATGTGTATAAGAGACAGGGACTACHVGGGTWTCTAAT).

154 Each genomic DNA dilution was amplified in triplicate. To test for bacterial DNA contamination 155 in PCR supplies, we included a "no template" control that used PCR grade water in place of 156 DNA as template. For a positive control, we used genomic DNA from a mock community of 20 157 bacterial strains (HM277-D, BEI Resources). PCR cycles were: $95^{\circ} \mathrm{C}$ for 3 minutes, then 25 158 cycles of $95^{\circ} \mathrm{C}$ for 30 seconds, $55^{\circ} \mathrm{C}$ for 30 seconds, and $72^{\circ} \mathrm{C}$ for 30 seconds, followed by a 159 final step of $72^{\circ} \mathrm{C}$ for 5 minutes. An aliquot of each reaction was analyzed by gel electrophoresis 160 to confirm presence of a band at the desired size. Equal volumes of each of the triplicate 161 reactions were pooled for each sample.

162

163 Pooled reactions were sent to the University of Rhode Island's Genomics and Sequencing Center, 164 where they were cleaned using $0.7 x$ Ampure XP beads. Cleaned reactions were quantified with a 165 Qubit analyzer, and approximately $50 \mathrm{ng}$ of DNA was used as template in the second 166 amplification step. The only exceptions to this were the negative kit control and the no template 167 control, which had low DNA concentration by Qubit analysis. For these, $5 \mu 1$ of the cleaned 168 reaction was used as template. Six cycles of amplification were performed using Phusion high169 fidelity PCR Mastermix and Nextera indices. Reactions were cleaned with 0.6x Ampure XP 
170 beads, quantified with a Qubit analyzer, and diluted to $10 \mathrm{nM}$. Three $\mu 1$ of each dilution were

171 pooled for the final library, along with $3 \mu 1$ of the reactions for the negative kit control and no

172 template control, which were less than $10 \mathrm{nM}$ based on Qubit analysis. Sequencing of the library

173 was performed on an Illumina MiSeq using a 500-cycle v2 kit.

174

175 Data analysis

176 16S rRNA amplicon data was analyzed using mothur v.1.36.1 (Schloss et al., 2009), following

177 the MiSeq SOP (https://www.mothur.org/w/index.php?title=MiSeq_SOP\&oldid=9609). To

178 assess positive and negative controls, all samples were analyzed together. Then, filter-digester

179 bed samples were analyzed separately to investigate changes in the bacterial community of the

180 digester over time, and liquid effluent samples were analyzed separately to compare sanitization

181 methods. In all analyses, sequences $<248$ bp or $>275$ bp and sequences with ambiguous bases

182 were discarded. Remaining sequences were aligned against Silva reference database release 123

183 (Yilmaz et al., 2014). Using UCHIME (Edgar et al., 2011), 2.8\% of sequences from filter-

184 digester bed samples and $3.1 \%$ of sequences from liquid effluent samples were identified as

185 chimeric and discarded. Remaining sequences were classified using the Ribosomal Database

186 Project 16S rRNA training set version 14 (Cole et al., 2014). For filter-digester bed samples, 42

187 sequences were classified as chloroplast, mitochondria, archaea, eukaryotic or unknown and

188 removed, whereas 179 sequences were removed during this step for liquid effluent samples.

189 Sequences were then classified into Operational Taxonomic Units (OTUs) at a 0.03 cutoff,

190 which means that sequences within an OTU are at least 97\% similar to each other. Filter-digester

191 bed samples ranged from 115,835 - 169,829 sequences, and each sample was subsampled to 
192 115,835 sequences. Liquid effluent samples ranged from 106,154 - 164,159 sequences, and each 193 sample was subsampled to 106,154 sequences.

194

195 Data and code

196 Raw MiSeq data, mothur output files and R code used for analyses can be found at the FigShare 197 repository:

198 https://figshare.com/projects/Data_and_R_code_for_Microflush_Composting_Toilet_Microbiom 199 e_Project/35342

200

201 Results

202 Sequencing of mock community detected all expected taxa with a low error rate, but not all taxa 203 classified to genus level

204 As a positive control, we sequenced a mock community (HM277-D, BEI Resources) alongside 205 the samples and negative controls. This mock community is comprised of known concentrations 206 of genomic DNA from 20 different bacterial species. In the mock community sample, we 207 detected 672 unique sequences from 181,020 total sequences. To estimate our overall error rate, 208 we compared these sequences to the known 16S rRNA gene sequences for the species 209 represented in the mock community. This gave an error rate of $0.006 \%$, which suggests that data 210 processing steps in mothur, such as removal of chimeras, were effective in reducing the error rate 211 for the sequences.

212

213 We then clustered the sequences into OTUs using a cutoff of $\geq 97 \%$ similarity. In the mock 214 community sample, there were 22 OTUs comprised of at least 10 sequences, and 89 OTUs 
215 comprised of $<10$ sequences. Of the 22 "abundant" OTUs, 18 were classified to the level of

216 genus, whereas two were only classified to the level of Family, and two were only classified to

217 the level of Order. Based on the expected representation in the mock community (Supplementary

218 Table 1), we matched each of the four OTUs classified to Family or Order with an expected

219 species (for example, Otu00046 was classified as Order Bacillales, and the mock community is

220 expected to include Bacillus cereus). We tested these matches by using BLAST to align a

221 representative sequence to the $16 \mathrm{~S}$ rRNA GenBank database. For each of the four OTUs,

222 BLAST analysis identified hits to the matched genus at $\geq 97 \%$ similarity. Overall, we identified

223 OTUs corresponding to each of the 20 expected mock community species. We did not identify

224 any unexpected OTUs comprised of $>10$ sequences; all of these "abundant" OTUs could be

225 matched to one of the species included in the mock community.

226

227 Analysis of the mock community indicates that sequencing and data processing accurately

228 identified OTUs, because all expected species were detected with a low error rate; however, it

229 revealed limitations in OTU classification, because some mock community OTUs could not be

230 classified to the level of genus. For filter-digester bed and liquid effluent samples, we addressed

231 these limitations by analyzing the data at different taxonomic levels, including detailed analysis

232 of "unclassified" OTUs.

233

234 Negative controls show minimal contamination of materials with bacterial DNA

235 We performed two types of negative controls to assess bacterial DNA contamination in

236 experimental materials. In the negative kit control to test genomic DNA extraction materials, we

237 detected 206 unique sequences from 1,123 total sequences, which were clustered into 184 OTUs. 
238 In the no template control to test PCR materials, we detected 37 unique sequences from 200 total

239 sequences, which were clustered into 34 OTUs. By comparison, the smallest number of total

240 sequences detected in a sample was 106,154. To further assess possible contamination of

241 materials with bacterial DNA, we determined how many unique sequences occurred in samples

242 and negative controls (Supplementary Figure 1). Ten unique sequences were detected in samples

243 (filter-digester bed only, liquid effluent only, or both) and both controls. Ten additional unique

244 sequences were detected in samples and the no template control, but not the kit control, whereas

245116 unique sequences were detected in samples and the kit control, but not the no template

246 control. For the kit control, some of these shared sequences may be due to cross-contamination

247 of the kit control with sample material, because the kit control was processed alongside samples.

248 Overall, only $1.1 \%$ of unique sequences detected in the filter-digester bed samples are also

249 detected in controls, and only $1.6 \%$ of unique sequences detected in the liquid effluent samples

250 are also detected in controls. This indicates that bacterial DNA contamination of materials should

251 have minimal impact on analysis and interpretation of sample data.

252

253 Bacterial taxa associated with deposition of fecal matter in test system are replaced over time

254 To understand how the composition of the bacterial community in the filter-digester bed changes

255 over time, we collected a sample immediately after depositing human fecal matter and then after

2564 , 10, 15, and 30 days, during which no additional materials were added. Each sample was

257 subsampled to 115,835 sequences. Analysis of filter-digester bed samples clustered the

258 subsampled sequences into 3,049 OTUs, which were classified into 24 phyla and one

259 "unclassified" group that includes all OTUs that could not be classified at the phylum level. Of

260 these 25 groups, seven phyla and the "unclassified" group occurred at a mean relative abundance 
$261>2 \%$ across all samples (Figure 2). None of the remaining 17 phyla occurred at a relative

262 abundance $>2 \%$ in any of the samples, and only Chloroflexi occurred at a relative abundance

$263>1 \%$ (in samples from day 10 and day 30 ).

264

265 At the level of phylum, the bacterial community of the time zero sample was dominated by

266 Firmicutes (70.0\%), followed by Bacteroidetes (26.8\%), Proteobacteria (2.5\%) and

267 Actinobacteria (0.3\%). By comparison, subsequent samples collected 4-30 days later showed a

268 shift in bacterial community composition in the filter-digester bed (Figure 2). The proportion of

269 Firmicutes decreased to $20.9 \%$ on day 4 and further decreased to $3.0 \%$ on day 10 . The proportion

270 of Bacteroidetes increased to $47.3 \%$ on day 4 , then decreased slightly but remained $>40 \%$

271 through day 30. Proteobacteria and Actinobacteria increased in abundance from time zero to day

2724 to day 10 and then remained stable. Beginning on day 4 , we detected three phyla that were not

273 present in the time zero sample: Verrucomicrobia, Acidobacteria and Planctomycetes. These

274 phyla were consistently detected in each of the filter-digester bed samples collected from day 4

275 through day 30.

276

277 To gain a finer scale perspective of the changes in bacterial community composition of the filter-

278 digester bed, we analyzed the OTUs at the level of genus. The 3,049 OTUs were classified into

279287 genera and one "unclassified" group that includes all OTUs that could not be classified at the

280 level of genus. The unclassified group comprised the largest proportion of sequences in each

281 sample and is discussed further below. In the time zero sample, ten genera occurred at a relative

282 abundance $>2 \%$, with $59.2 \%$ of sequences clustering into these groups (Figure $3 a$ ). The genera

283 belonged to either Bacteroidetes or Firmicutes. For each genus except Clostridium XI, relative 
284 abundance decreased over the subsequent samples until it was $<0.02 \%$ in the day 30 sample. By

285 contrast, the proportion of Clostridium XI remained above $0.8 \%$ in each sample through day 30.

286

287 In the samples collected between day 4 and day 30, new genera were detected that were not

288 observed in the time zero sample (Figure 3b). In particular, six genera occurred at a mean

289 relative abundance $>2 \%$ across these samples but did not occur in the time zero sample. These

290 genera belong to Actinobacteria, Bacteroidetes, Proteobacteria and Verrucomicrobia. This genus-

291 level analysis suggests that the increase in abundance of Bacteroidetes, Proteobacteria and

292 Actinobacteria over the sampling period was due to a change in the composition of genera in the

293 filter-digester bed rather than an increase in abundance of the same genera observed at time zero.

294

295 Because the "unclassified" group included such a large proportion of sequences in each sample,

296 and because analysis of the mock community suggested that some "standard" taxa may not be

297 fully classified to the genus level in our data, we analyzed unclassified OTUs on their own.

298 Overall, this group comprised $35.6 \%$ of sequences in the time zero sample. This proportion

299 decreased to $26.5 \%$ in the day 4 sample before increasing over the subsequent samples to $62.3 \%$.

300 To investigate this further, we analyzed individual unclassified OTUs. Relative abundance in the

301 following analysis refers to the abundance of a particular unclassified OTU compared to the total

302 number of sequences clustered into the unclassified group during the genus level analysis. In the

303 time zero sample, six OTUs occurred at a relative abundance $>2 \%$ and included $78.7 \%$ of the

304 total sequences in the unclassified group (Supplementary Figure 2a). Each of these OTUs

305 decreased in abundance in the subsequent samples until they occurred at $<0.15 \%$ in the day 30

306 sample. Beginning with the day 4 sample, new unclassified OTUs were detected. In particular, 
307 seven OTUs occurred at a mean relative abundance $>2 \%$ across the samples collected on and

308 after day 4 (Supplementary Figure 2b). These OTUs included $23.5-46.4 \%$ of the sequences in the

309 unclassified group of each sample; however, none of them were detected in the time zero sample.

310 These data parallel the genus level data and further illustrate a shift in the bacterial community

311 composition of the filter-digester bed over time after fecal matter was deposited.

312

313 To compare the overall bacterial community structure of the filter-digester bed over the time

314 series, we used two different beta diversity metrics: Bray-Curtis dissimilarity, which accounts for

315 abundance of OTUs but not their phylogenetic relatedness, and weighted UniFrac distance,

316 which assesses phylogenetic relatedness of OTUs and weights branches based on OTU

317 abundance. Dendrograms of the resulting distance matrices showed that samples from the last

318 three time points cluster together (Supplementary Figure 3). The Bray-Curtis distance matrix

319 clustered the day 4 sample with the time zero sample, albeit with a large distance between these

320 two samples, whereas the weighted UniFrac distance matrix clustered the day 4 sample with the

321 last three samples. Overall, beta diversity analysis provides additional evidence of a major shift

322 in the bacterial community composition of the filter-digester bed occurring within a few days

323 after deposition of fecal matter.

324

325 Bacterial taxa detected in untreated liquid effluent are more abundant in SODIS-treated effluent

326 but greatly reduced or absent from SSF-treated effluent

327 To characterize the bacterial community of the liquid effluent from the test system, we collected

328 a sample of effluent on day 15 from a drainage pipe connected to the filtrate chamber at the base

329 of the test system. A portion of this sample was analyzed as "untreated" liquid effluent. We used 
330 the remainder to test two different sanitization methods: Solar Disinfection (SODIS) and Slow

331 Sand Filter (SSF). A sample of the output from each method was analyzed as "treated" liquid

332 effluent. Each of the effluent samples was subsampled to 106,154 sequences.

333

334 Analysis of the liquid effluent samples clustered the subsampled sequences into 2,903 OTUs, 335 which were classified into 24 phyla and one "unclassified" group that included all OTUs that 336 could not be classified at the phylum level. Seven of these phyla and the unclassified group

337 occurred at a relative abundance $>2 \%$ in at least one of the effluent samples (Figure 4).

338 Proteobacteria was the dominant phylum in all three samples, comprising $45.0 \%$ of sequences in 339 the untreated effluent, $56.9 \%$ of sequences in the SODIS-treated effluent, and $39.9 \%$ of 340 sequences in the SSF-treated effluent. Bacteroidetes was abundant in the untreated and SODIS341 treated effluent (36.1\% and $39.2 \%$ of sequences, respectively), but this phylum comprised only

$3423.7 \%$ of sequences in the SSF-treated effluent. By contrast, the unclassified group and

343 Verrucomicrobia occurred at a higher relative abundance in the SSF-treated effluent compared to

344 the untreated and SODIS-treated effluent. The other four phyla occurred at a relative abundance $345 \leq 5 \%$ in all liquid effluent samples.

346

347 To further examine the differences in bacterial community composition between untreated and 348 treated liquid effluent, we analyzed the OTUs at the level of genus. Eleven genera and an 349 "unclassified" group occurred at a relative abundance $>2 \%$ in at least one of the samples. The 350 untreated effluent and both treated effluents were dominated by the unclassified group, which 351 comprised $48.9-76.0 \%$ of sequences and is further discussed below. Five genera

352 (Flavobacterium, Hyphomonas, Algoriphagus, Hydrogenophaga, and Roseomonas) that 
353 occurred at a relative abundance $>2 \%$ in the untreated effluent were detected at a higher relative

354 abundance in the SODIS-treated effluent (Figure 5). By contrast, these genera were not

355 prominent members of the SSF-treated effluent community, occurring at a relative abundance

$356<0.4 \%$. In addition, two minor members of the untreated effluent community (Methylophilus and

357 Rheinheimera) increased in abundance in the SODIS-treated effluent. The abundance of

358 Methylophilus was unchanged in SSF-treated effluent. We detected only one sequence classified

359 as Methylophilus in the negative controls, therefore we can conclude that the persistence of

360 Methylophilus is not an artifact of contamination. In the SSF-treated effluent, three genera that

361 occurred at low relative abundance in the untreated and SODIS-treated effluent (Ancylobacter,

362 Planctomyces, and Opitutus) were detected at a higher abundance.

363

364 Because many of the sequences in our genus level analysis were clustered into an "unclassified"

365 group, we analyzed these OTUs separately. Relative abundance in the following analysis refers

366 to the abundance of a particular unclassified OTU compared to the total number of sequences

367 clustered into the unclassified group during the genus level analysis. Fourteen unclassified OTUs

368 occurred at a relative abundance $>2 \%$ in at least one of the three effluent samples

369 (Supplementary Figure 4). These OTUs accounted for 61.7\% of total sequences in the

370 unclassified group for untreated effluent, $89.7 \%$ for SODIS-treated effluent and $43.5 \%$ for SSF-

371 treated effluent.

372

373 Of the seven unclassified OTUs that occurred at a relative abundance $>2 \%$ in the untreated

374 effluent, four of these increased in abundance in the SODIS-treated effluent, paralleling the trend

375 observed at the genus level analysis. In addition, two OTUs that occurred at a relative abundance 
$376 \quad 1-2 \%$ in the untreated effluent also increased in abundance in the SODIS-treated effluent. All six

377 of these OTUs were minor members of the SSF-treated effluent, occurring at a relative

378 abundance $<0.08 \%$. The SSF treatment appeared to introduce OTUs into the effluent. Five OTUs

379 that occurred in the SSF-treated effluent were either not detected in the untreated and SODIS-

380 treated effluent or detected only at a low relative abundance $(0.03-0.07 \%)$. The abundance of one

381 OTU (Otu0012) was largely consistent across the liquid effluent samples. We detected only eight

382 sequences classified into this OTU in the negative controls, therefore we can conclude that the

383 persistence of this OTU is not an artifact of contamination.

385 Comparing the bacterial community composition of the untreated liquid effluent to that of the 386 filter-digester bed sample also collected on day 15, we found only two genera (Flavobacterium 387 and Algoriphagus) at a relative abundance $>2 \%$ in both the liquid effluent and filter-digester bed 388 samples. This suggests that the bacterial community of the liquid effluent from the test system is 389 different from that of the solid filter-digester bed.

392 Discussion

393 Based on analysis of OTUs at different taxonomic levels and beta diversity metrics, a major shift 394 in the bacterial community composition of the filter-digester bed at the site of fecal matter 395 deposition occurs within a few days. The phylum-level profile of the time zero sample is 396 consistent with that of the human gut microbiome (Hugon et al., 2015; Turnbaugh et al., 2007), 397 which is expected because this sample was collected immediately after depositing human fecal 398 matter. At the genus level, the bacterial community of the time zero sample is dominated by 
399 known human gut microbes such as Bacteroides, Blautia and Faecalibacterium (Franzosa et al.,

400 2014; Rajilić-Stojanović and de Vos, 2014). With the exception of Clostridium XI, these gut-

401 associated genera are detected at extremely low relative abundance $(<0.02 \%)$ in the filter-

402 digester bed at day 30, and most are already greatly reduced by day 4 . By contrast, Clostridium

403 XI was detected in the time zero sample and all subsequent samples. This group includes the

404 gastrointestinal pathogen C. difficile and other Clostridium species, which are anaerobic spore-

405 forming bacteria (Pérez-Cobas et al., 2014).

406

407 The ability to withstand harsh environmental conditions over extended periods of time by

408 forming spores likely explains why Clostridium XI does not disappear from the filter-digester

409 bed by day 30. Persistence of spore-forming Clostridium species has been observed in other

410 studies of composting toilets (Tønner-Klank et al., 2007), and these species are resistant to

411 chemical treatment of human waste with urea (Vinnerås et al., 2003). Treatment of human waste

412 with peracetic acid successfully eliminated spore-forming Clostridium species (Vinnerås et al.,

413 2003); however, application of this chemical may not be practical or desirable for communities

414 using the GSAP Microflush toilet. Not all spore-forming bacteria are associated with disease,

415 therefore detection of Clostridium XI does not necessarily indicate a threat to public health from

416 compost material, but this result highlights the importance of considering persistence of certain

417 species via spore formation in the filter-digester bed.

418

419 In the subsequent samples collected beginning on day 4 from the site of initial fecal matter

420 deposition, taxa were detected that did not appear in the time zero sample, including three new

421 phyla and six new genera. These taxa persisted through to the day 30 sample. The six genera are 
422 primarily associated with environmental sources rather than the human gut microbiome. For

423 example, Flavobacterium and Algoriphagus belong to Bacteroidetes and have been isolated from

424 soil and aquatic environments (Nedashkovskaya et al., 2007; Thomas et al., 2011).

425 Pseudoxanthomonas and Rhodobacter belong to Proteobacteria, which increased in abundance

426 over the time series. Pseudoxanthomonas has been found as a dominant taxon in maize stover

427 composted by a process using the earthworm E. fetida (Chen et al., 2015), which are present in

428 the filter-digester bed studied here. These taxa detected beginning on day 4 were likely already

429 established in the filter-digester bed as a result of ongoing maintenance by periodic addition of

430 vegetable scraps.

431

432 The data indicate that the composition of the filter-digester bed at the site of deposition of fecal

433 matter shifts from a bacterial community dominated by taxa associated with the human gut to

434 taxa associated with the filter-digester bed itself. The conditions of the filter-digester bed may

435 not be amenable to gut-associated taxa, since many gut bacteria are anaerobes, and the GSAP

436 Microflush toilet is designed to use an aerobic composting process. In addition, bacterial taxa

437 that have become established within the filter-digester bed may outcompete gut-associated taxa

438 that are periodically introduced. In both the test system studied here and the field system

439 deployed in a community setting, the exact composition of the bacterial community of the filter-

440 digester bed will be influenced by how the GSAP Microflush toilet system is maintained and

441 used; for example, how often and what kind of non-fecal material is added to the filter-digester

442 bed. Using the test system as an indicator of the performance of the field system, we have shown

443 how the GSAP Microflush toilet can be used to immediately contain and over time eliminate gut- 
444 associated bacteria, thereby improving sanitation and contributing to better public health in rural

445 and peri-urban communities.

446

447 Typically, liquid effluent from the GSAP Microflush toilet exits the system into a soak hole that

448 acts as a leach field. However, this design is not suitable for communities with high water tables

449 due to concerns about contamination of water sources. In these situations, alternative sanitization

450 strategies are needed. Here, we compared the effects of solar disinfection (SODIS) and slow sand

451 filtration (SSF) on liquid effluent from the test system. Bacterial genera that were abundant in the

452 untreated liquid effluent on day 15 are generally associated with environmental sources such as

453 mud and freshwater. Flavobacterium and Algoriphagus were detected in both the filter-digester

454 bed sample collected on day 15 and the untreated liquid effluent collected on the same day,

455 suggesting that bacteria in the filter-digester bed may enter the filtrate chamber via liquid

456 flowing through the screens and mesh separating these two compartments. The untreated liquid

457 effluent also included Hyphomonas, which is typically associated with marine environments. Its

458 presence may be related to the test system's location on the coast of Narragansett Bay.

459

460 The genera and unclassified OTUs that were abundant in the untreated liquid effluent were

461 greatly reduced or not detected in the SSF-treated effluent; however, these taxa increased in

462 abundance in the SODIS-treated effluent. A previous study of SODIS for application with the

463 GSAP Microflush toilet system showed significant reduction of viable E. coli over eight hours of

464 exposure to sunlight (Mecca et al., 2013b). Given this and other studies showing the capacity of

465 SODIS to sanitize contaminated water (Boyle et al., 2008; Caslake et al., 2004), it is possible that 
466 bacterial cells in the SODIS-treated effluent are no longer viable, but DNA is recoverable and

467 detected using the 16S rRNA gene sequencing methods applied here.

468

469 In contrast to SODIS, the physical filtration approach of SSF treatment resulted in extremely low

470 or undetectable levels of most genera and unclassified OTUs found in the untreated liquid

471 effluent. This is expected, because this sanitization method is designed to physically remove

472 bacterial cells from the liquid effluent. We noted that SSF-treated effluent included taxa that

473 were not detected in either the untreated effluent or the SODIS-treated effluent. These taxa are

474 likely introduced from the schmutzdecke, which is a naturally formed biological layer of

475 microbes and invertebrates that is the key component of SSF treatment.

476

\section{Conclusions}

478 Overall, analysis of both the filter-digester bed and the liquid effluent of the GSAP Microflush

479 toilet test system illustrated the capacity of the design to isolate and eliminate bacteria associated

480 with human waste, thereby improving sanitation, contributing to the maintenance of safe water

481 sources and protecting public health. The invertebrate-enhanced aerobic composting process

482 reduces fecal-associated taxa in the filter-digester bed within a few days, with the exception of

483 spore-forming Clostridium species that are detected at low abundance even after 30 days. In

484 addition, the data presented here provide further insight towards the design of alternative

485 strategies for sanitization of liquid effluent in communities with high water tables.

486

487 Acknowledgements 
488 We thank Janet Atoyan at the University of Rhode Island Genomics and Sequencing Center for

489 assistance with 16S rRNA amplicon sequencing. We thank Kyle Edmonds and Lauren Precopio

490 for valuable contributions to establishing protocols for sample processing and data analysis. We

491 are grateful to Jeff Hollister, Pat Schloss and Nicole Sukdeo for advice regarding data analysis.

492

493 Funding

494 This research was supported by funds from Providence College, including a grant from the

495 Committee on Aid to Faculty Research. This material is based upon work conducted at a Rhode

496 Island NSF EPSCoR research facility, the Genomics and Sequencing Center, supported in part

497 by the National Science Foundation EPSCoR Cooperative Agreement \#EPS-1004057.

498

499

\section{References}

500 Boyle, M., Sichel, C., Fernández-Ibáñez, P., Arias-Quiroz, G.B., Iriarte-Puña, M., Mercado, A., 501 Ubomba-Jaswa, E., and McGuigan, K.G. (2008). Bactericidal Effect of Solar Water Disinfection 502 under Real Sunlight Conditions. Appl. Environ. Microbiol. 74, 2997-3001.

503 Caporaso, J.G., Lauber, C.L., Walters, W.A., Berg-Lyons, D., Lozupone, C.A., Turnbaugh, P.J., 504 Fierer, N., and Knight, R. (2011). Global patterns of 16S rRNA diversity at a depth of millions of 505 sequences per sample. Proc. Natl. Acad. Sci. 108, 4516-4522.

506 Caslake, L.F., Connolly, D.J., Menon, V., Duncanson, C.M., Rojas, R., and Tavakoli, J. (2004). 507 Disinfection of Contaminated Water by Using Solar Irradiation. Appl. Environ. Microbiol. 70, 508 1145-1150.

509 Chen, Y., Zhang, Y., Zhang, Q., Xu, L., Li, R., Luo, X., Zhang, X., and Tong, J. (2015).

510 Earthworms modify microbial community structure and accelerate maize stover decomposition 511 during vermicomposting. Environ. Sci. Pollut. Res. 22, 17161-17170.

512 Cole, J.R., Wang, Q., Fish, J.A., Chai, B., McGarrell, D.M., Sun, Y., Brown, C.T., Porras-Alfaro, 513 A., Kuske, C.R., and Tiedje, J.M. (2014). Ribosomal Database Project: data and tools for high 514 throughput rRNA analysis. Nucleic Acids Res. 42, D633-642.

515 Edgar, R.C., Haas, B.J., Clemente, J.C., Quince, C., and Knight, R. (2011). UCHIME improves 516 sensitivity and speed of chimera detection. Bioinformatics 27, 2194-2200. 
517 Elliott, M.A., Stauber, C.E., Koksal, F., DiGiano, F.A., and Sobsey, M.D. (2008). Reductions of 518 E. coli, echovirus type 12 and bacteriophages in an intermittently operated household-scale slow 519 sand filter. Water Res. 42, 2662-2670.

520 Franzosa, E.A., Morgan, X.C., Segata, N., Waldron, L., Reyes, J., Earl, A.M., Giannoukos, G., 521 Boylan, M.R., Ciulla, D., Gevers, D., et al. (2014). Relating the metatranscriptome and 522 metagenome of the human gut. Proc. Natl. Acad. Sci. 111, E2329-E2338.

523 Hijnen, W. a. M., Schijven, J.F., Bonné, P., Visser, A., and Medema, G.J. (2004). Elimination of 524 viruses, bacteria and protozoan oocysts by slow sand filtration. Water Sci. Technol. 50, 147-154.

525

526

527

528

529

530

531

532

533

534

535

536

537

538

539

540

541

542

543

544

545

546

547

548

549

550

551

552

553

Hill, G.B., and Baldwin, S.A. (2012). Vermicomposting toilets, an alternative to latrine style microbial composting toilets, prove far superior in mass reduction, pathogen destruction, compost quality, and operational cost. Waste Manag. 32, 1811-1820.

Hugon, P., Dufour, J.-C., Colson, P., Fournier, P.-E., Sallah, K., and Raoult, D. (2015). A comprehensive repertoire of prokaryotic species identified in human beings. Lancet Infect. Dis. $15,1211-1219$.

Mecca, S., Davis, H., and Davis, A. (2013a). Application of GSAP Microflush toilets: a sustainable development approach to rural and peri-urban sanitation. In WIT Transactions on Ecology and the Environment, (WIT Press), pp. 113-122.

Mecca, S., Pellock, B., and Bretz, R. (2013b). Pathogen removal options: emphasizing SOLDIS for the filtrate of a GSAP Microflush toilet. In WIT Transactions on Ecology and the Environment, (WIT Press), pp. 201-213.

Mecca, S., Stifler, C., and Beley, C. (2017). Influence of Temperature on the Spatial Distribution of Macro-Organisms in the GSAP Microflush Toilet Digester and On Extended Applications of the Technology. J. Phys. Sci. Environ. Stud. 3, 1-7.

Nedashkovskaya, O.I., Kim, S.B., Kwon, K.K., Shin, D.S., Luo, X., Kim, S.-J., and Mikhailov, V.V. (2007). Proposal of Algoriphagus vanfongensis sp. nov., transfer of members of the genera Hongiella Yi and Chun 2004 emend. Nedashkovskaya et al. 2004 and Chimaereicella Tiago et al. 2006 to the genus Algoriphagus, and emended description of the genus Algoriphagus Bowman et al. 2003 emend. Nedashkovskaya et al. 2004. Int. J. Syst. Evol. Microbiol. 57, 1988-1994.

Pérez-Cobas, A.E., Artacho, A., Ott, S.J., Moya, A., Gosalbes, M.J., and Latorre, A. (2014). Structural and functional changes in the gut microbiota associated to Clostridium difficile infection. Front. Microbiol. 5, 335.

Rajilić-Stojanović, M., and de Vos, W.M. (2014). The first 1000 cultured species of the human gastrointestinal microbiota. FEMS Microbiol. Rev. 38, 996-1047.

Schloss, P.D., Westcott, S.L., Ryabin, T., Hall, J.R., Hartmann, M., Hollister, E.B., Lesniewski, R.A., Oakley, B.B., Parks, D.H., Robinson, C.J., et al. (2009). Introducing mothur: Open-Source, Platform-Independent, Community-Supported Software for Describing and Comparing Microbial Communities. Appl. Environ. Microbiol. 75, 7537-7541. 
554 Soobhany, N., Mohee, R., and Garg, V.K. (2017). Inactivation of bacterial pathogenic load in 555 compost against vermicompost of organic solid waste aiming to achieve sanitation goals: A 556 review. Waste Manag. 64, 51-62.

557 Thomas, F., Hehemann, J.-H., Rebuffet, E., Czjzek, M., and Michel, G. (2011). Environmental 558 and Gut Bacteroidetes: The Food Connection. Front. Microbiol. 2.

559 Tønner-Klank, L., Møller, J., Forslund, A., and Dalsgaard, A. (2007). Microbiological 560 assessments of compost toilets: In situ measurements and laboratory studies on the survival of 561 fecal microbial indicators using sentinel chambers. Waste Manag. 27, 1144-1154.

562 Turnbaugh, P.J., Ley, R.E., Hamady, M., Fraser-Liggett, C., Knight, R., and Gordon, J.I. (2007). 563 The human microbiome project: exploring the microbial part of ourselves in a changing world.

564 Nature 449, 804-810.

565 UN General Assembly (2015). Transforming our world: the 2030 agenda for sustainable 566 development.

567 Vinnerås, B., Holmqvist, A., Bagge, E., Albihn, A., and Jönsson, H. (2003). The potential for 568 disinfection of separated faecal matter by urea and by peracetic acid for hygienic nutrient 569 recycling. Bioresour. Technol. 89, 155-161.

570 World Health Organization (2018). World health statistics 2018: monitoring health for the SDGs, 571 sustainable development goals.

572 World Health Organization, and United Nations Children's Fund (2017). Progress on drinking 573 water, sanitation and hygiene: 2017 update and SDG baselines.

574 Yilmaz, P., Parfrey, L.W., Yarza, P., Gerken, J., Pruesse, E., Quast, C., Schweer, T., Peplies, J., 575 Ludwig, W., and Glöckner, F.O. (2014). The SILVA and "All-species Living Tree Project 576 (LTP)" taxonomic frameworks. Nucleic Acids Res. 42, D643-D648. 
Figure 1 (on next page)

Diagram of GSAP Microflush toilet test system

When used in a community setting, the toilet is enclosed and includes a small hand wash station. 
Microflush Valve

Top Front Slab

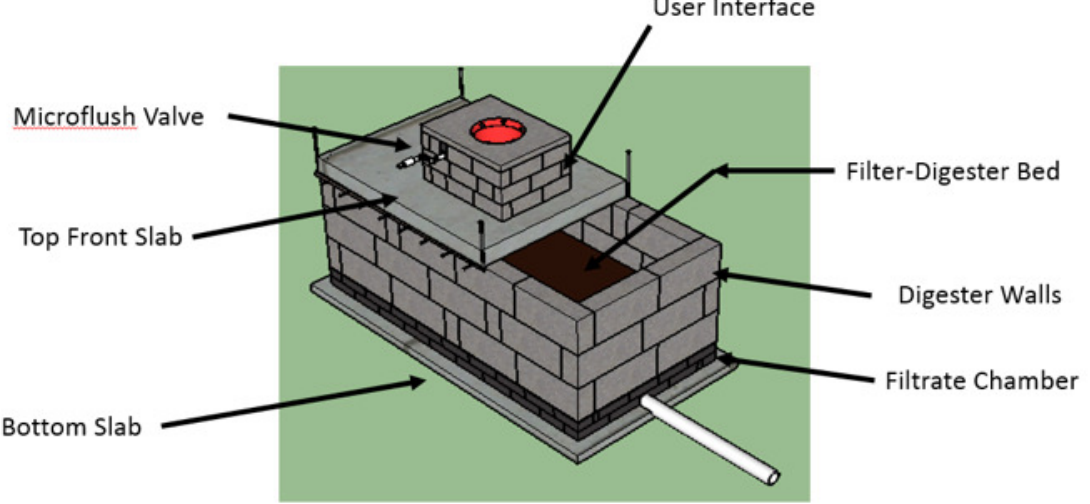




\section{Figure 2 (on next page)}

Relative abundance of phylum-level groups occurring at a mean relative abundance $>2 \%$ across all filter-digester bed samples. 


\section{Figure 3 (on next page)}

Relative abundance of genera occurring in filter-digester bed samples.

A. Genera occurring at $>2 \%$ in the time zero sample, shown for all filter-digester bed samples. B. Genera occurring at a mean relative abundance $>2 \%$ in the four subsequent samples (day 4 - day 30), shown for all filter-digester bed samples. 
Figure 4 (on next page)

Relative abundance of phylum-level groups occurring at $>2 \%$ in at least one of the liquid effluent samples. 


\section{Figure $\mathbf{5}$ (on next page)}

Relative abundance of genera occurring at $>2 \%$ in at least one of the liquid effluent samples. 


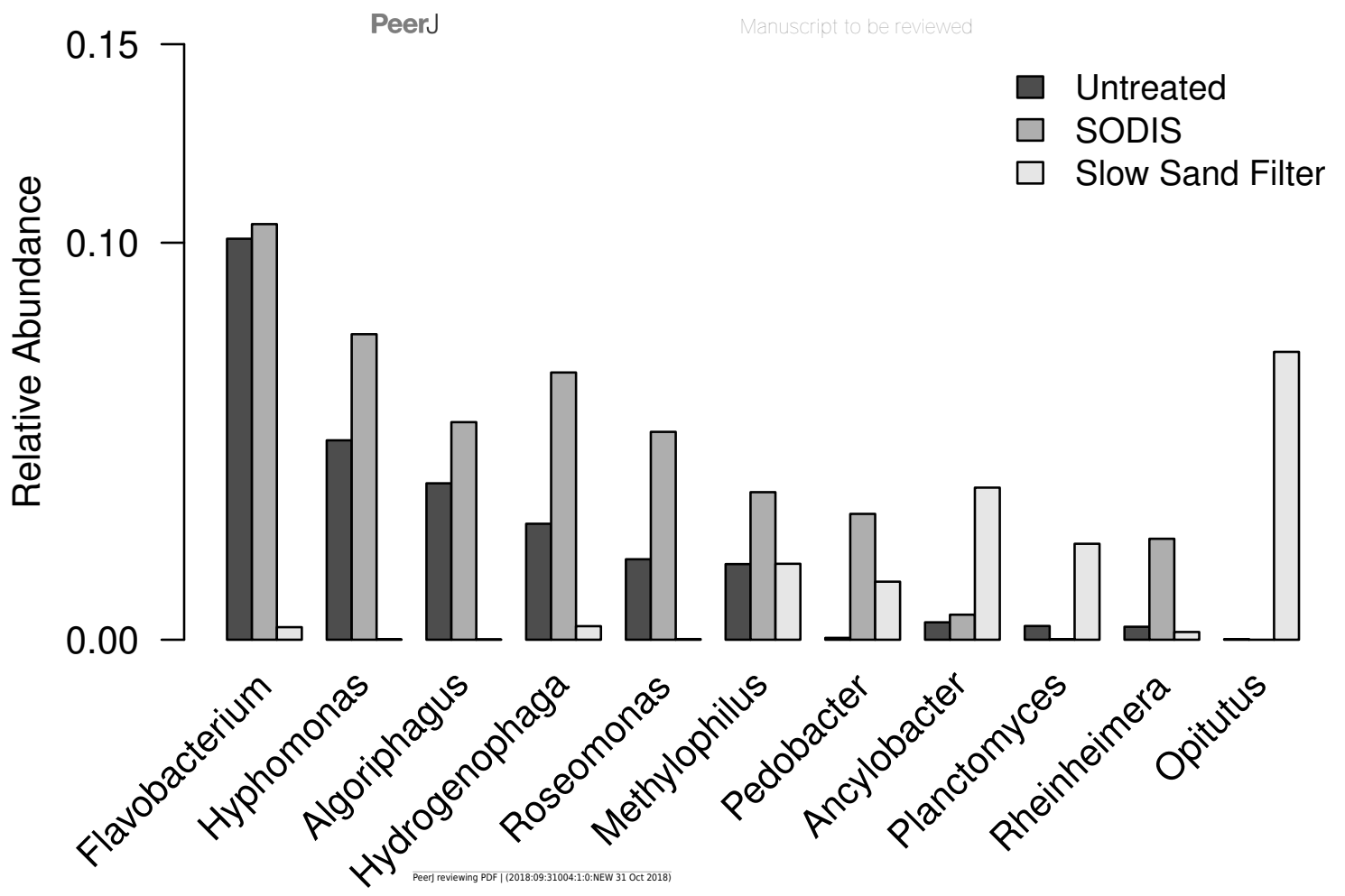

